\title{
GRAINE 2018 experiment: Performance evaluation of gamma-ray telescope utilizing nuclear emulsion
}

\author{
Yuya Nakamura* for the GRAINE Collaboration ${ }^{\dagger}$ \\ Nagoya University \\ E-mail: ynakamuradflab.phys.nagova-u.ac.70
}

\begin{abstract}
The observation of cosmic gamma-rays is crucial to understanding high-energy astrophysical phenomena. The Gamma-Ray Astro-Imager with Nuclear Emulsion (GRAINE) aims to precisely observe gamma-ray sources with a balloon-borne gamma-ray telescope utilizing nuclear emulsion, which is a three-dimensional tracking detector with a sub-micron spatial resolution. We launched the third balloon experiment, GRAINE 2018, on April 26, 2018 as a demonstration project. The flight was successful, data acquisition was completed, and we are analyzing the data now. We evaluated the angular resolution for gamma-rays in the $100-300 \mathrm{MeV}$ energy region of the converter's flight data, and it is $0.57^{\circ}(\tan \theta \simeq$ zenith angle $<0.5), 0.81^{\circ}(0.5<\tan \theta<1.0)$. This is better than the required performance value, which is $1.0^{\circ}$ in $100-\mathrm{MeV}$ energy region.
\end{abstract}

The 4th KMI International Symposium (KMI2019)

18-20, February 2019

Nagoya, Japan

${ }^{*}$ Speaker.

${ }^{\dagger}$ Nagoya University, Kobe University, Okayama University of science, Aichi University of education, ISAS/JAXA 


\section{Introduction}

The observation of cosmic gamma-rays is crucial to understanding high-energy astrophysical phenomena such as black holes, pulsars, supernova remnants and so on. The Gamma-Ray Astro-Imager with Nuclear Emulsion (GRAINE) is a balloon-borne gamma-ray telescope project utilizing nuclear emulsion[四]. Nuclear emulsion is a three-dimensional tracking detector with submicron spatial resolution, and it can precisely determine gamma-ray angles by measuring electron and positron tracks via pair production $\left(\gamma \rightarrow \mathrm{e}^{+}+\mathrm{e}^{-}\right)$just below the conversion point. Its angular resolution for gamma-rays, estimated by using Monte Carlo simulation and evaluated with accelerator beam tests, is approximately one order of magnitude higher than that of Fermi-LAT, which is the latest sub-GeV/GeV gamma-ray detector launched in 2008[प, [ ]]. Furthermore, it can measure the azimuthal angle of the plane in which the electron and positron tracks lie, and it has sensitivity to gamma-ray polarization [B], 团]. GRAINE aims to precisely observe gamma-ray sources with high angular resolution and polarization sensitivity in the $10 \mathrm{MeV}$ to $100 \mathrm{GeV}$ energy region with a large aperture area $\left(10 \mathrm{~m}^{2}\right)$.

To date, we have repeated balloon experiments and developed the detector. Most recently, was our third balloon experiment, GRAINE 2018, was conducted on April 26, 2018[[]]. The aim was to detect the brightest gamma-ray source, the Vela pulsar, in the $100-\mathrm{MeV}$ energy region with an angular resolution of $<1.0^{\circ}$ for the demonstration. The flight was successful, data acquisition was completed, and data analysis is now in progress.

The main components of the emulsion gamma-ray telescope are a converter, a time stamper, and a star camera. The gamma-ray angle and energy are determined in the converter. For GRAINE 2018 , it consists of 100 stacked emulsion films, each comprised of a $180-\mu \mathrm{m}$-thick plastic film and $75-\mu$ m-thick emulsion layers applied on both sides measuring $25 \times 38 \mathrm{~cm}^{2}$. The total amount of substance is 0.52 radiation lengths. In GRAINE2018, four converters are mounted, and the total aperture area is $\sim 0.38 \mathrm{~m}^{2}$. The time stamper provides the arrival time of each track by using a multistage shifter[6], and the star camera provides the attitude of the telescope during the observation. Therefore, each event can be mapped in celestial coordinates by combining angle, time, and attitude information.

In this paper, we will report on the evaluation of gamma-ray angular resolution in the converter.

\section{Evaluation of the angular resolution by analyzing the hadronic interactions}

\subsection{Evaluation method}

The left illustration in Figure $\mathbb{W}$ shows the evaluation method. Primary cosmic rays enter the converter during the observation. Some cause hadronic interactions and produce $\pi^{0}$ particles. Each $\pi^{0}$ decays into two gamma-rays just near the interaction points, so the angle of the gamma-ray is estimated by connecting the hadronic interaction point, which is almost the same as the $\pi^{0}$ decay point, and the conversion point to the electron pair. Therefore, the angular resolution in the converter is given by the following equations:

$$
\begin{gathered}
\sigma \tan \theta_{1}=\sqrt{(\sigma \Delta \tan \theta)^{2}-\left(\sigma \tan \theta_{2}\right)^{2}}, \\
\Delta \tan \theta=\tan \theta_{2}-\tan \theta_{1}
\end{gathered}
$$


where $\tan \theta_{1}$ is the angle determined by measuring the electron pair tracks and $\tan \theta_{2}$ is the estimated angle found by connecting the two points. The right panel in Figure $\mathbb{U}$ shows the definition of the angle. The $\mathrm{Z}$ axis is defined as the thickness direction of the film, the $\mathrm{R}$ axis is defined as the running direction of the estimated gamma-ray, and the $\mathrm{L}$ axis is defined as the direction orthogonal to the $\mathrm{R}$ axis.

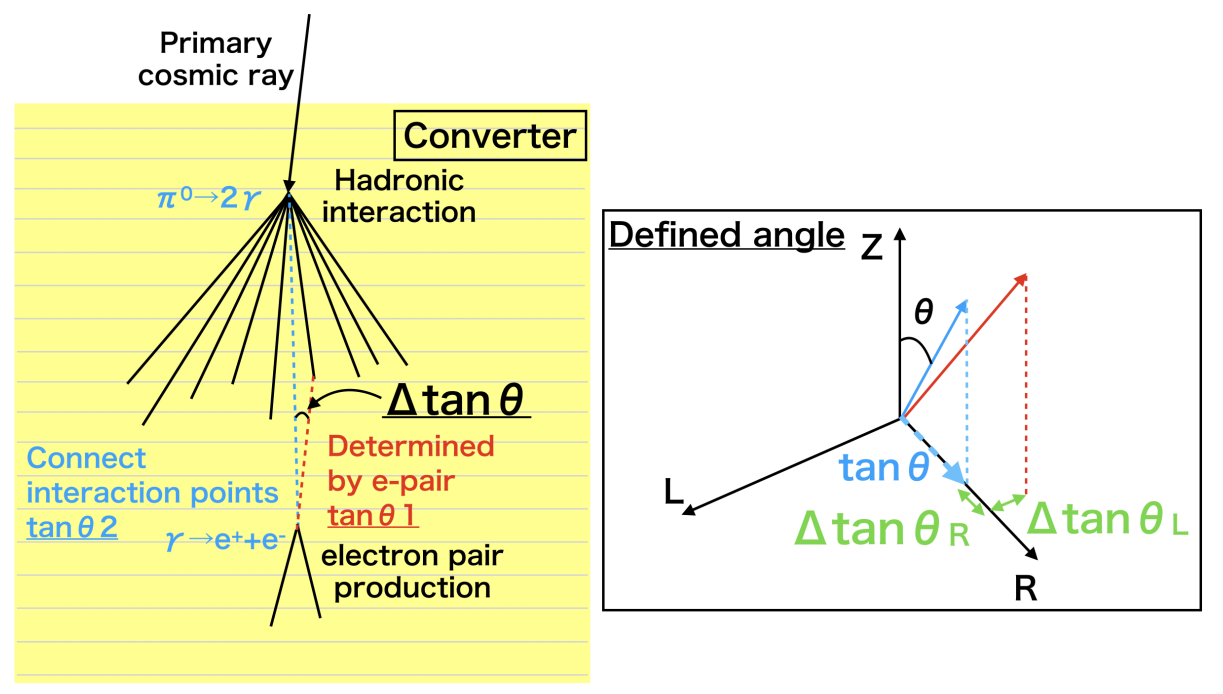

Figure 1: left: Concept of the evaluation method. right: Definition of the angle.

\subsection{Dataset}

Seven films are used for continuously selecting the hadronic interaction events in the converter. The fourth film is defined as the search film. Tracks connecting with the first film, the most upstream film, and not penetrating the three downstream films are rejected in the search film. The remaining tracks are extrapolated to the upstream direction, and, if some of them converge within the second and third films, they are defined as the daughter tracks of the hadronic interaction. The distance between the interaction point and the extrapolated point of the daughter tracks to the same $\mathrm{R}-\mathrm{L}$ plane is defined as the parameter of how they converge, and it is called the minimum distance. We used the hadronic interactions for this analysis if the number of daughter tracks were $>10$ and the average of the minimum distance was $<10 \mu \mathrm{m}$. In total 10,634 events were selected in the 50 upstream films of the two units of the converter.

Gamma-ray events were selected by using the method developed for the GRAINE 2015, the second balloon experiment $[\square]$. We used events win the 100-300 MeV energy region, and 1,131,594 events were selected in the 80 downstream films of the two units of the converter.

\subsection{Results and discussion}

Figure 2 displays the distribution of $\Delta \tan \theta$ after subtracting the random background in the $\tan \theta_{1}<0.5$ angular range. This is made by randomly shifting the hadronic interaction points within the size of the film while maintaining the $\mathrm{Z}$ position. $\Delta \tan \theta_{\mathrm{R}, \mathrm{L}}=0$ is in the highest bin and the bin width is $0.01 \times 0.01$, which means that the hadronic interaction points are imaged by detected 
gamma-rays as expected. Figure 3 presents the projected distribution made by summing the bin entries in $-0.025<\Delta \tan \theta_{\mathrm{LorR}}<+0.025$ for the $\mathrm{R}$ and $\mathrm{L}$ components respectively. $\sigma \Delta \tan \theta$ is defined as its Gaussian-fitted sigma, and, in this angular range, $\sigma \Delta \tan \theta_{\mathrm{R}} \simeq 0.0087 \pm 0.0020, \sigma \Delta \tan \theta_{\mathrm{L}} \simeq$ $0.0095 \pm 0.0011$. $\sigma \tan \theta_{2(\mathrm{R}, \mathrm{L})}$ is evaluated by using high momentum tracks that penetrated the converter from top to bottom with negligible scattering. $\sigma \tan \theta_{1(\mathrm{R}, \mathrm{L})}$ is calculated by using equation 2.1 and converted to $\sigma \theta_{1(\mathrm{R}, \mathrm{L})}$ by the following equation:

$$
\sigma \theta_{1(\mathrm{R}, \mathrm{L})}= \pm \arctan \left(\tan \theta_{1(0-0.5)} \pm \sigma \tan \theta_{1(\mathrm{R}, \mathrm{L})}\right) \mp \arctan \left(\tan \theta_{1(0-0.5)}\right)
$$

where $\tan \theta_{1(0-0.5)}$ is the weighted average angle of the gamma-rays used for Figure 3 and the sign is used conservatively to be $>\sigma \theta_{1(\mathrm{R}, \mathrm{L})}$. The results give $\sigma \theta_{1 \mathrm{R}}=0.36 \pm 0.11^{\circ}$ and $\sigma \theta_{1 \mathrm{~L}}=$ $0.44 \pm 0.06^{\circ}$. Therefore, we evaluated all the tracks in the $0.5<\tan \theta_{1}<1.0$ angular range by using the same method, and Table 1 presents all the results. The angular resolution is defined as $\sigma \theta_{1}=\sqrt{\sigma \theta_{1 \mathrm{R}}^{2}+\sigma \theta_{1 \mathrm{~L}}^{2}}$, and it is $0.57^{\circ}(0<\tan \theta<0.5)$ and $0.81^{\circ}(0.5<\tan \theta<1.0)$ using this calculation, which is better than the required performance $\left(1.0^{\circ}\right.$ in the $100-\mathrm{MeV}$ range).

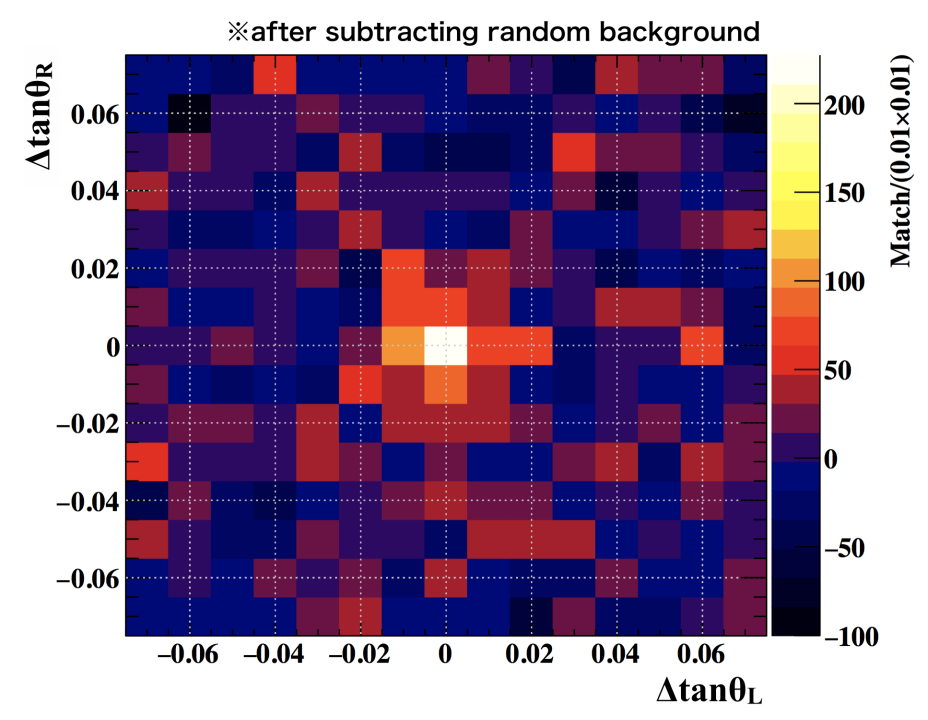

Figure 2: Distribution of the $\Delta \tan \theta$ after subtracting the random background

\begin{tabular}{|c|c|c|c|}
\multicolumn{3}{c}{ preliminary } \\
\hline $\tan \theta$ & $\sigma \theta_{1 \mathrm{R}}$ & $\sigma \theta_{1 \mathrm{~L}}$ & $\sigma \theta_{1}$ \\
\hline $0-0.5$ & $0.36^{\circ}$ & $0.44^{\circ}$ & $0.57^{\circ}$ \\
\hline $0.5-1.0$ & $0.72^{\circ}$ & $0.37^{\circ}$ & $0.81^{\circ}$ \\
\hline
\end{tabular}

Table 1: The results of the evaluation of the angular resolution in the converter. $\sigma \theta_{1}=\sqrt{\sigma \theta_{1 \mathrm{R}}^{2}+\sigma \theta_{1 \mathrm{~L}}^{2}}$ 

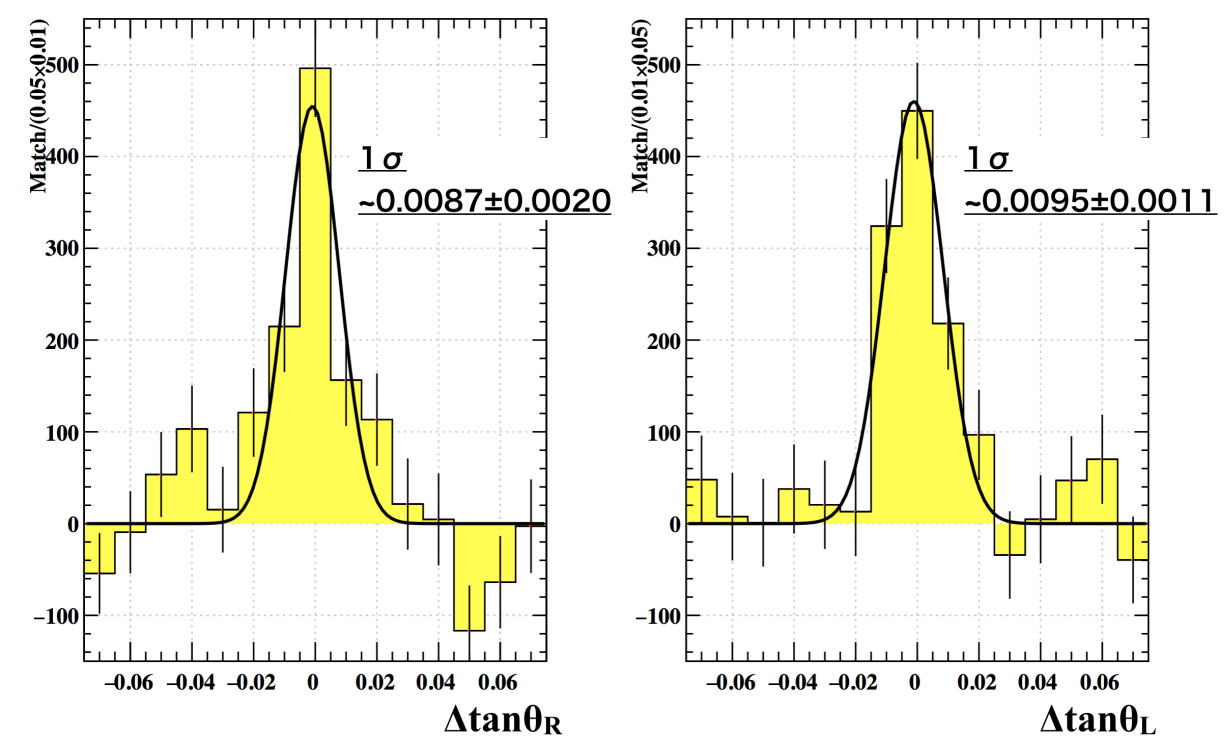

Figure 3: Projected distribution of the $\Delta \tan \theta_{\text {RorL }}$.

\section{Summary and future prospect}

We are promoting the GRAINE project to realize high-angular-resolution, polarization-sensitive cosmic gamma-ray observation with a balloon-borne gamma-ray telescope utilizing a nuclear emulsion, which has an extremely high spatial resolution. GRAINE's third balloon experiment (GRAINE 2018) was launched in April 2018 for the demonstration. We evaluated the angular resolution of the converter used in GRAINE 2018 with the angular dependence by analyzing the gamma-rays produced from hadronic interactions. With the present evaluation results, the angular resolution is estimated as $0.57^{\circ}(0<\tan \theta<0.5)$ and $0.81^{\circ}(0.5<\tan \theta<1.0)$ in the $100-300 \mathrm{MeV}$ energy region. This is better than the required performance $\left(1.0^{\circ}\right.$ in the $100-\mathrm{MeV}$ range), demonstrating that the telescope worked as expected during the observation. Combined analysis (using the converter, time stamper, and attitude monitor) is ongoing to detect the Vela pulsar.

\section{References}

[1] S. Takahashi et al., Adv. Space Res. 62, 2945 (2018).

[2] W. B. Atwood et al., Astrophys. J. 697, 1071 (2009).

[3] S. Takahashi, PhD thesis, Nagoya University, Japan (2011) (in Japanese).

[4] K. Ozaki et al., Nucl. Instrum. Methods A 833, 165 (2016).

[5] S. Aoki et al., RADIOISOTOPES (in Japanese, submitted).

[6] S. Takahashi et al., Nucl. Instrum. Methods A 620, 192 (2010).

[7] H. Rokujo et al., Prog. Theor. Exp. Phys. 2018, 063 H01 (2018). 